\title{
Cutaneous Leishmaniasis with Long Duration and Bleeding Ulcer
}

Marco Manfredi ${ }^{1,2^{*}}$, Silvia luliano ${ }^{1}$, Barbara Bizzarri², Alessandro Fugazza ${ }^{2}$, Pierpacifico Gismondi ${ }^{1}$ and Gian Luigi de'Angelis ${ }^{1,2}$

${ }^{1}$ Department of Pediatrics, Azienda Ospedaliero-Universitaria di Parma, University Hospital-Parma-Italy

${ }^{2}$ Gastroenterology and Endoscopy Unit, Azienda Ospedaliero-Universitaria di Parma, University Hospital-Parma-Italy

"Corresponding author: Marco Manfredi, Department of Pediatrics, Azienda Ospedaliero-Universitaria di Parma, University Hospital-Parma-Italy, Tel: +393472822003; Fax: +39-521-702647; E-mail: marco.manfredi8@gmail.com

Received date: October 16, 2015; Accepted date: January 07, 2016; Published date: January 14, 2016

Copyright: @ 2016 Manfredi M, et al. This is an open-access article distributed under the terms of the Creative Commons Attribution License, which permits unrestricted use, distribution, and reproduction in any medium, provided the original author and source are credited.

Abstract
Leishmaniasis may cause visceral, cutaneous and/or mucocutaneous diseases.
Cutaneous and mucocutaneous forms are caused by a single celled parasite transmitted by sand fly bites.
Although the cutaneous form of the disease is often self-limiting, it results in significant scarring and can spread to
more invasive, mucocutaneous disease. Therefore, treatment may be considered to prevent these complications.
We describe a case report of cutaneous leishmaniasis contracted in a healthy man in Italy (Emilian Apennines).
This cutaneous ulcer healed only with intralesional injection of meglumine antimoniate.
After about 18 and 30 months a scar area is still present and no satellite lesion appeared.
We have had no side effects or complications due to therapy.

\section{Introduction}

Leishmaniasis comprises a number of diseases of the viscera, skin and/or mucous membranes.

This disease is transmitted by several genera and species of sand flies.

Three major clinical forms of leishmaniasis are recognized: a systemic leishmaniasis caused by L. donovani, the old world cutaneous leishmaniasis caused by L. major, L. tropica and L. aethiopica and the new world or american leishmaniasis caused by $L$. mexicana and $L$. braziliensis. Leishmania is an obligate intracellular parasite that in vertebrate hosts, exists only in the amastigote stage [1].

The transmission occurs by contamination from the site of sand flies injection. Many of the promastigotes inoculated do not survive for direct cytolytic action by the tissues of mammals. Some promastigotes are phagocytized by histiocytes-macrophages, become amastigotes and thus begin replicating. The main areas at risk of infection are the Mediterranean, the Middle East and parts of Asia. Leishmania tropica causes self-limiting skin ulcers in which the parasites are found within macrophages at the edge of the lesion.

Dogs and rodents are often the natural reservoir of infection. There are two types of old world leishmaniasis: the wet and dry type.

The wet type is predominantly rural and is caused by L. major, it has a short incubation time, few parasites into the lesion and a fast recovery [2].

On the contrary, the dry type is a rather urban anthropo-zoonoses caused by L. tropica. It has a long incubation period, a long duration of infection and many parasites within the dermis of the lesion.
In the early phase, there is a layer of corneal hypertrophy and hyperplasia of the dermal papillae resulting in necrosis of the central area due to obstruction papillary.

The ulcer becomes depressed with hardened and thickened edges and friable granulation tissue at the base. At this time it can be confused with carcinoma, tuberculous lesion, and syphilitic nodule.

Cutaneous leishmaniasis (CL) may be limited to a single part of the skin or may produce multiple lesions [1].

Histologically there are lymphocytes, plasma cells, epithelioid cells and large giant cells.

After the phlebotomus bite, an itchy red papulovesicular lesion appears; after some weeks or months, the surface of the papule dries, the crust is formed and then it falls and gives rise to the deep ulcer.

Although the cutaneous form of the disease is often self-limiting, it does result in significant scarring and can spread to more invasive, mucocutaneous disease. Therefore, treatment may be considered to prevent these complications $[3,4]$.

Topical therapies to treat CL include both pharmacologic and nondrug modalities. Non-drug therapies including cauterization, surgical excision, cryotherapy, but the simplest of these treatments seems to be the local application of heat [3].

The WHO recommends intralesional or systemic antimonials for the treatment of CL $[1,2,5]$.

Intralesional infiltration with pentavalent antimony produces the maximum concentration in the lesion and has few systemic side effects, but does not reach the metastatic injuries [3]. 
Citation: Manfredi M, luliano S, Bizzarri B, Fugazza A, Gismondi P, et al. (2016) Cutaneous Leishmaniasis with Long Duration and Bleeding Ulcer. Clin Microbiol 5: 229. doi:10.4172/2327-5073.1000229

Page 2 of 5

The recommended therapy of localized CL is an injection of 1-3 $\mathrm{ml}$ of pentavalent antimony under the edges of the lesion and under the entire lesion until the surface has blanched. This infiltration should be performed every 5-7 days for a total of 2-5 times [6].

Intralesional meglumine antimoniate also proved effective in a Sardinian study [7].

\section{Case Report}

A male of 45 years old were simultaneously bitten by many sand flies (there were counted about 150 inocula) mainly to bilateral shins. About 2-3 days later appeared subsequent local allergic reaction (edema, warmth) mimicking cellulitis associated to inconstant and low-grade fever (Figure 1).

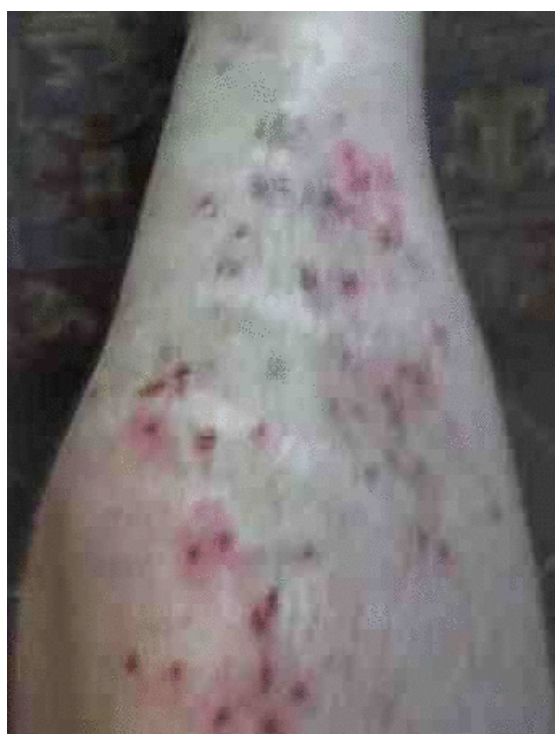

Figure 1: Local allergic reaction mimicking cellulitis.

He had gone to the emergency room where he was treated with steroids and antihistamines (the first doses by intramuscular injection and then orally) for a total of seven days with moderate efficacy and improvement of the lesion.

After a few months, the lesion on the right shin, of about $5 \mathrm{~cm}$ in diameter, became round, psoriasiform (Figure 2); scarifying the centre, the lesion showed exudative and bleeding aspect (Figure 3).

The lesion has always been totally asymptomatic (no itching, no pain, no fever).

It is characterized by large central ulcerated region, friable and excavated with bloody bottom with hypertrophic and hyperemic slightly scaly edges (Figure 3 ).

He was initially treated with topical antibiotics (first with gentamicin, then retapamulin) with apparent initial moderate improvement. As a result of acute sinusitis, the patient took oral penicillin protected with apparent temporary improvement of the ulcer.
A first dermatologist performed biopsies which showed granulomatous tissue of likely infectious origin; the search of bacteria, parasites, fungi and acid-alcohol resistant bacteria was negative.

The cultural examination of the ulcer bottom showed no specific pathogens (except for the presence of $S$. epidermidis).

The patient has never done steroids (excepted the initial doses due to allergic reaction) or immunosuppressive drugs and he does not have immune deficiencies; he was a blood donor until the ulcer appearance. In recent years he has not gone abroad (his last trip was 5 years earlier to Paris).

Further tests (anti-rickettsia, anti-borrelia, anti-bartonella, and antileishmania antibodies, VDRL, and the Mantoux test) were negative. The basic parameters showed a moderate rise in the $\mathrm{C}$ Reactive Protein.

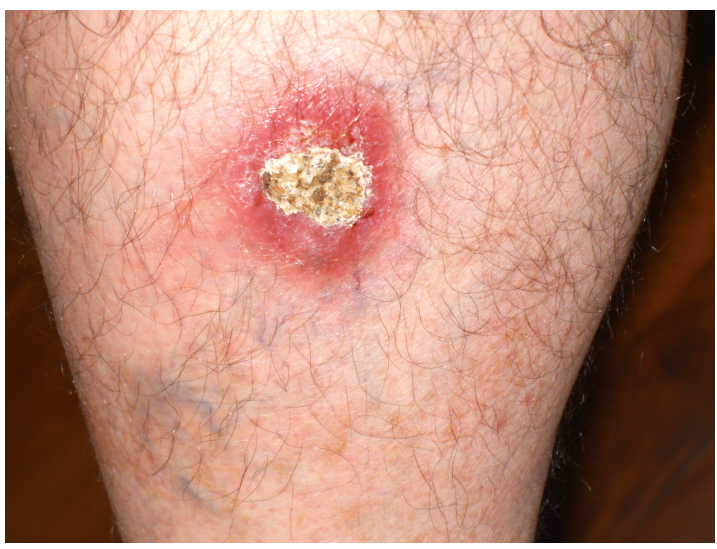

Figure 2: Lesion on the right shin.

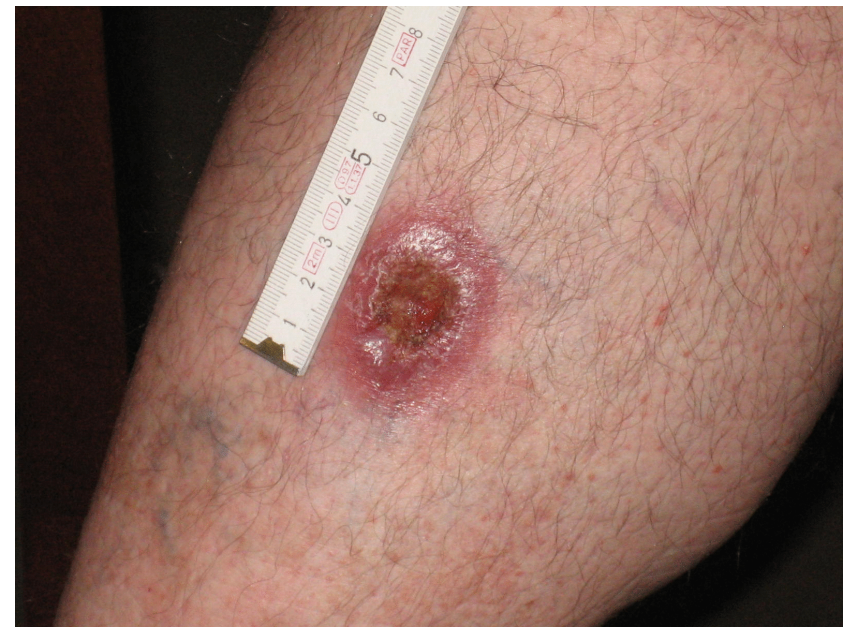

Figure 3: Lesion showing exudative and bleeding aspect. 
Citation: Manfredi M, luliano S, Bizzarri B, Fugazza A, Gismondi P, et al. (2016) Cutaneous Leishmaniasis with Long Duration and Bleeding

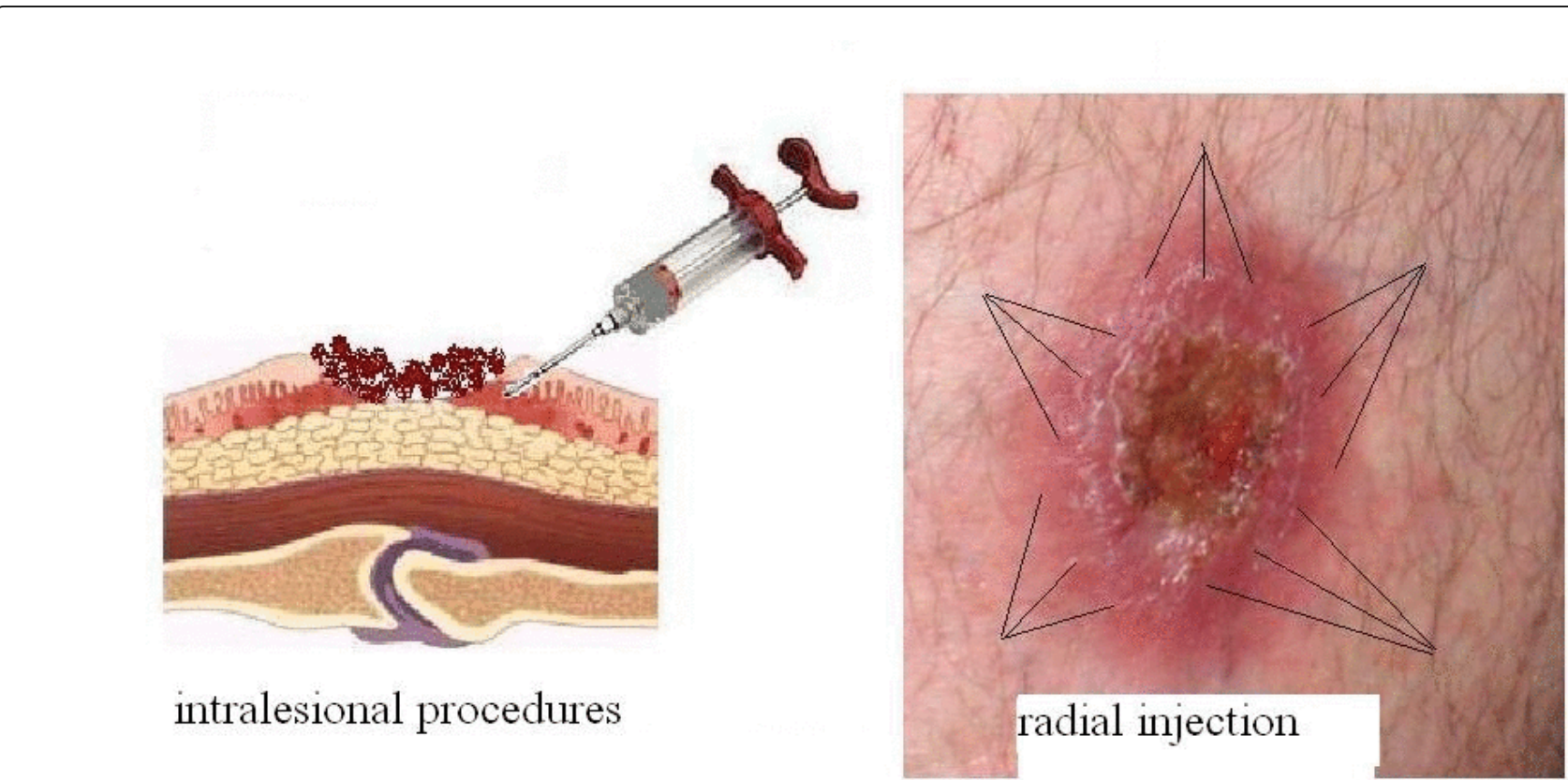

Figure 4: Drug injection around the lesion.

He repeated biopsy several months later, after our clinical suspicion of leishmaniasis; the histological exam showed mild epidermal hyperplasia with intense lymphoplasmacytic infiltrate of the superficial and intermediate dermis. Within this infiltrate, several outbreaks of granulomatous inflammation (known as "tuberculoid") containing Langhans type giant cells and some small area of dermal necrosis are present. Nor fungi with PAS or specific staining, neither acid-alcohol resistant bacilli were shown. No evidence of organisms related to protozoa, also sought by staining with giemsa.

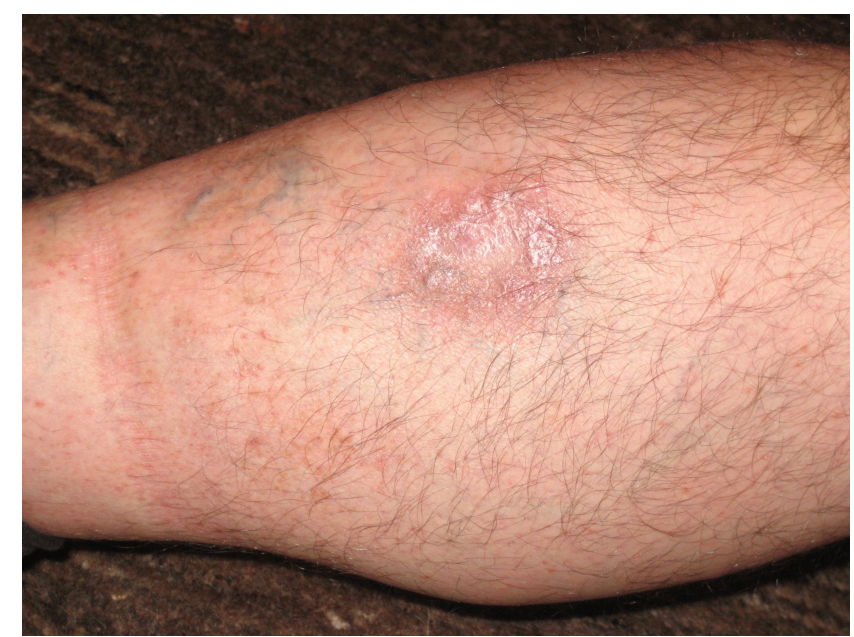

Figure 5: Dry lesion.

The pathologist concluded that the morphological picture observed may be more consistent with cutaneous leishmaniasis, in an appropriate clinical context.
The dermatologist recommended intralesional therapy with meglumine antimoniate or surgical excision. Considering the good tolerability of treatment described in the literature, we preferred to perform the first hypothesis.

Five sessions were performed by injecting of about 3-4 $\mathrm{ml}$ of drug each time to 7 days apart from each other. The injection was executed to obtain the whitening of the skin area overlying the inoculation, as described by Blum. It should infiltrate the area around the lesion including the base of the ulcer with a fine needle (25G) into the derma. During the injections (Figure 4), the patient complained of moderate pain and heat sensation at the injection site, quickly disappeared.

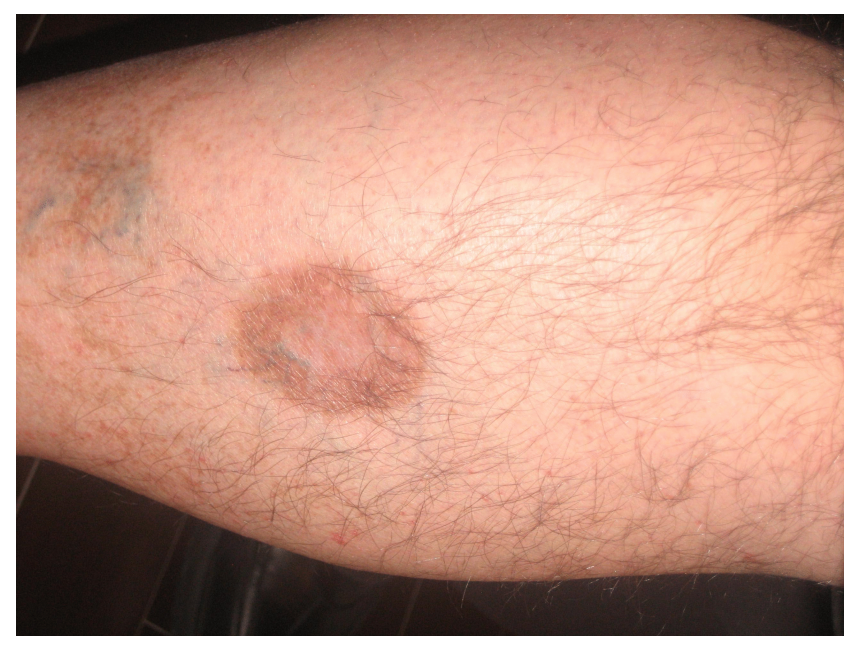

Figure 6: Flat scar appearance. 
After the first three sessions, the ulcer showed initial regression of exudative-necrotic central area and of peripheral hyperemia. After about 2 weeks after the last injection, the lesion appeared dry and flat, slightly scaly (Figure 5).

There were no side effects during and after injections.

After about 18 months, the lesion was epithelialized, with flat scar appearance (Figure 6). The same pattern was present a year later.

\section{Discussion}

Tuscan-Emilian Apennine is considered endemic area of zoonoses by Leishmania, especially among dogs and deer, although human cases are very sporadic as hygienists of patient's health district have confirmed. Old world leishmania not necessarily induce mucocutaneous infection.

Probably the patient contracted the parasite after numerous and simultaneous sand fly bites which resulted in increase of pathogen load with subsequent development of the lesion.

The isolated sand fly bites that occurring frequently during summer does not transmit a parasitic loads that cause lesions; this patient has a very clear and sensible skin also to mosquito bites that may have favored this massive attack by sand flies. Administration of steroids after initial local allergic reaction, perhaps, may have contributed to the high load of parasite.

Although CL is not a life-threatening disease, and it is often selflimiting, it is very annoying and can result in significant scarring; moreover it can spread to more invasive mucocutaneous disease. Therefore, treatment may be considered to prevent these complications [3].

We believe that the parasite has not been shown histologically for two reasons: in the first test, because the biopsy was performed only on the surface, without clinical suspicion.

The second time, with a clinical suspicion of leishmaniasis, but several months had passed away after injection and probably the lesion began to self-restraint.

However, the pathologist's conclusion was clear: compatible with leishmaniasis in the appropriate clinical setting. The clinic was characteristic and other causes have been excluded (tuberculosis, syphilis, mycosis). Laboratory exams such as anti-leishmania antibodies have variable sensitivity and specificity and are often negative in cutaneous form [2].

Probably this lesion had features of both types; wet (rural, likely a few parasites) and dry type (anthropo-zoonoses, long period of incubation, long duration of skin ulcer) of old world leishmaniasis.

Intralesional administration is an attractive option: the dosages of the drug are much lower thus minimizing potentially systemic side effects, the times of action are rapid, the high concentrations of drug saturate active lesions, the costs are lower and the complications like scarring are reduced [5].

Intralesional infiltration with antimoniates causes the maximum concentration in the lesion and has rare systemic side effects $[6,8]$. Several pentavalent antimonials are available currently: sodium stibogluconate and its generic forms, used in field conditions, and meglumine antimoniate. Intramuscular or intravenous antimony has been used in visceral leishmaniasis for years.
The world health organization (WHO) recommendations for the treatment of cutaneous leishmaniasis are intralesional or systemic antimonials, according to the species and the clinical features. Local infiltration with pentavalent antimony has been used in Old World localized cutaneous leishmaniasis. WHO recommends an injection of $1-3 \mathrm{ml}$ under the edges of the lesion and the entire lesion until the surface has blanched. The infiltration could be given every 5-7 days, for a total of 2-5 times. In 1999, a systematic review of the reported series showed that intra-lesional antimonials partially or completely cured $72-97 \%$ of the lesions caused by L. major. More recently, 106 pediatric cutaneous leishmaniasis cases have been published in Tunisia. Species typing is not mentioned in the series. However, lesions are supposed to be due to $L$. major, which is endemic in Tunisia, or to L. infantum which is isolated sporadically in Northern Tunisia, where most of the children lived. In the latter study, intra-lesional meglumine antimoniate $\left(1 \mathrm{ml} / \mathrm{cm}^{2} /\right.$ week, until recovery) was used in children with fewer than five lesions, and/or without lesion on a cartilaginous area (ear, nose), or $63 \%$ of the subjects. The average number of injections for cure is 1.9, but some patients have been treated for one year. Pain during injection is reported frequently by the children. In another study in this country, including 272 children or adults treated with intra-lesional injections, adverse events were observed in 5\%: bacterial super-infections (especially when cutaneous leishmaniasis was located on face or limbs), stibio-intolerance signs (in cephalic locations) or evolution to sporotrichoi"d lesions. In $L$. major cutaneous leishmaniasis, intra-lesional injections of meglumine antimoniate have been compared to intramuscular injections in a randomized study. There was no statistically significant difference between the two groups on day 30. Intra-lesional antimonials seem to be less effective in $L$. tropica cutaneous leishmaniasis. More than 3000 cases, presumed to be due to this species, have been reported in Turkey. $66 \%$ of the patients were under 19 years. Intra-lesional injections of meglumine antimoniate $\left(1 \mathrm{ml} / \mathrm{cm}^{2}\right)$ were used in $76 \%$ of the patients regardless of the age. The average number of injections was 11 . With a prolonged treatment however, relapses are reported less frequently than with short courses (4-6\%) [1].

The drug must not be injected into the subcutaneous tissue because it would be rapidly absorbed and does not reach the site of infection.

The drug must be injected under the edges of the lesion and the entire lesion until the surface has blanched; two to five injections every 5-7 days [6].

Intralesional antimoniates is an effective agent against old world CL. It is to be reserved to mild and few lesions, it involves very few side effects and it can be useful in outpatient setting [9].

\section{References}

1. Minodier P, Parola P (2007) Cutaneous leishmaniasis treatment. Travel Med Infect Dis 5: 150-158.

2. Reithinger R, Dujardin JC, Louzir H, Pirmez C, Alexander B, et al. (2007) Cutaneous leishmaniasis. Lancet Infect Dis 7: 581-596.

3. Palumbo E (2010) Treatment Strategies for Mucocutaneous Leishmaniasis. J Glob Infect Dis 2: 147-150.

4. Clem A (2010) A Current Perspective on Leishmaniasis. J Glob Infect Dis 2: 124-126.

5. Moskowitz PF, Kurban AK (1999) Treatment of cutaneous leishmaniasis: retrospective and advances for the 21st century. Clin Dermatol 17: 305-315. 
Citation: Manfredi M, luliano S, Bizzarri B, Fugazza A, Gismondi P, et al. (2016) Cutaneous Leishmaniasis with Long Duration and Bleeding Ulcer. Clin Microbiol 5: 229. doi:10.4172/2327-5073.1000229

Page 5 of 5

6. Blum J, Desjeux P, Schwartz E, Beck B, Hatz C (2004) Treatment of cutaneous leishmaniasis among travellers. J Antimicrob Chemother 53: 158-166.

7. Aste N, Pau M, Ferreli C, Biggio P (1998) Intralesional treatment of cutaneous leishmaniasis with meglumine antimoniate. Br J Dermatol 138: 370-371.
8. Masmoudi A, Maalej N, Boudaya S, Turki H, Zahaf A (2006) Adverse effects of intralesional Glucantime in the treatment of cutaneous leishmaniasis. Med Mal Infect 36: 226-228.

9. Solomon M, Baum S, Barzilai A, Pavlotsky F, Trau H, et al. (2009) Treatment of cutaneous leishmaniasis with intralesional sodium stibogluconate. J Eur Acad Dermatol Venereol 23: 1189-1192. 